\title{
Le nouvel écosystème web développé par une chaîne de service public. Le cas de France 2
}

Article inédit. Mis en ligne le 23 décembre 2013.

\section{Laurence Leveneur}

Laurence Leveneur est maître de conférences en Sciences de l'Information et de la Communication à l'Université de Toulouse 1 et à l'IUT de Rodez. Membre de l'IDETCOM (Institut du Droit, de l'Espace, des Territoires et de la Communication) (Université de Toulouse 1 Capitole) et membre associé du CEISME (Centré d'Etudes sur les Images et les Sons médiatiques (Sorbonne Nouvelle Paris 3), elle a publié plusieurs articles portant notamment sur les jeux et les divertissements télévisuels et les stratégies des marques télévisuelles sur les réseaux socio-numériques.

Plan

Service public de l'audiovisuel et logiques du web dynamique.

L'extension de la marque France 2 à l'écosystème web.

Conclusion

Références bibliographiques

\section{Résumé}

Tandis que les chaînes de télévision investissent chaque jour davantage l'univers numérique, personne ne semble d'accord sur les spécificités d'un service public de l'audiovisuel en ligne. En quoi le développement de l'offre numérique du groupe France Télévisions diffère-t-il des logiques qui gouvernent ses concurrents privés? Nous souhaiterions, dans cet article, tenter de répondre à ces questions par une analyse sémiopragmatique du site internet et des comptes ouverts sur Twitter et Facebook par France 2, principale chaîne du groupe public. Nous complèterons cette étude par une observation ethnographique des forums de discussions de son site internet. Nous confronterons ainsi l'offre numérique développée France 2, hors téléphonie mobile, aux discours stratégiques des acteurs du groupe.

Mots clés: Service public, Audiovisuel public, Internet, Réseaux socio-numériques, sémiologie, ethnographie.

\section{Abstract}

While television channels invest more each day the digital world, nobody seems to agree on the specifics of a public service broadcasting online. How the development of digital services of France Télévisions group differs from the logic governing its private competitors? We would, in this article, try to answer these questions in a pragmatic semiotic analysis of website and accounts on Twitter and Facebook by France 2, the main channel of the public group. We will complete this study with an ethnographic observation of its websites' forums. Thus, we will compare the numeric development of France 2 to its leaders' speeches.

Keywords: Public Service, Public Broadcasting, Internet, digital social networks, semiotics, ethnography. 


\section{Resumen}

Si bien la televisión invierten cada día más al mundo digital, nadie parece ponerse de acuerdo sobre los detalles de un servicio público de radiodifusión en línea. Cómo el desarrollo de los servicios digitales del grupo France Télévisions difiere de la lógica que rige sus competidores privados? Nosotros, en este artículo, tratamos de responder a estas preguntas en un análisis semiótico pragmática de sitio web y cuentas en Twitter y Facebook por France 2, el canal principal del grupo público. Vamos a completar este estudio con una observación etnográfica de los foros de discusión de su sitio web. Y vamos a comparar el ofrece digital de France 2 con los discursos de sus lideres.

Palabras clave: Servicio Público, para la Difusión Pública, Internet, las redes sociales digitales, la semiótica, la etnografía.

\section{INTRODUCTION}

Plusieurs auteurs ont souligné la labilité de l'expression «service public». Le vocable semble surchargé de significations spécifiques, notamment dans le contexte européen et français. Jacques Chevallier a observé à quel point l'expression avait connu, d'une façon générale, un ébranlement profond au cours du XXème siècle (Chevallier, 2007), la logique d'action du service public n'apparaissant plus comme étant radicalement opposée à celle du privé, désormais soumise aux mêmes contraintes d'efficacité. Quelques principes demeurent cependant, comme le «rôle social » qui lui est attribué : accessible à tous, il apparaît comme un agent de redistribution devant réduire les inégalités sociales et « (...) répondre aux aspirations de tous ordres des individus et des groupes.» (Chevallier, $2007: 16)$

L'auteur rappelle que son régime juridique spécifique s'articule autour de trois principes : continuité, égalité et mutabilité (Chevallier, 2007:16). Ce dernier suppose que les règles de fonctionnement d'un service public s'adaptent à toute époque, et notamment aux nouvelles technologies de l'information et de la communication. L'arrivée du web a ainsi conduit plusieurs administrations publiques à développer leurs offres numériques, en particulier au sein des collectivités territoriales (Voir Laurent, Marchandise, 2013). Cette volonté est d'autant plus prégnante s'agissant de l'audiovisuel public, comme l'attestent les récents rapports de Jean-Pierre Plancade (Plancade, 2013) ou de Pierre Lescure (Lescure, 2013), soulignant tout deux les enjeux que représente « (...) la diminution de l'importance de la primo-diffusion par voie hertzienne » (Plancade, 2013: 46) au profit de l'audience différée (télévision de rattrapage, vidéo à la demande, paiement par abonnement, etc.).

Mais tandis que les chaînes de télévision investissent chaque jour davantage l'univers numérique, personne ne semble d'accord sur les spécificités d'un service public de l'audiovisuel numérique. Le fameux triptyque « informer, cultiver, distraire » reste au cœur des discours et des promesses des dirigeants de l'audiovisuel public qui défendent, depuis plus de cinquante ans, le pluralisme et la diversité des programmes. Rémy Pflimlin, PDG du groupe France Télévisions, trace la frontière entre télévision privée et télévision publique en soulignant "l'utilité sociale» de cette dernière (Revel, 2011). Rassembler le plus grand nombre de téléspectateurs serait aussi le gage d'une télévision de service public qui doit, selon les mots de son dirigeant, être un « (...) espace de compréhension et non un espace de consommation» (Revel, 2011). Si, comme le souligne Bruno Patino, Directeur général délégué aux programmes, aux antennes et aux développements numériques, «(...) plus 
personne ne peut considérer que la mission du service public se limite à mettre en ligne les émissions de télé » (Lherm, Charpentier, 2011), en quoi le développement de l'offre numérique du groupe public diffère-t-il des logiques qui gouvernent ses concurrents privés? Les équipes digitales des chaînes privées et publiques ne prônent-elles pas finalement les mêmes valeurs, à savoir des échanges personnalisés et le développement de nouvelles façons de consommer du contenu audiovisuel ? Le groupe France Télévisions estil véritablement en capacité d'innover et d'entreprendre sur le web dynamique une démarche favorisant l'égalité d'accès et la promotion des offres culturelles numériques?

Nous souhaiterions, dans cet article, tenter de répondre à ces questions par une analyse sémio-pragmatique du site internet et des comptes ouverts sur Twitter et Facebook par France 2, principale chaîne généraliste du groupe audiovisuel public ${ }^{1}$. Nous complèterons cette étude par une observation ethnographique des forums de discussions de son site web (concernant la méthodologie employée, voir Barats, coord., 2013, pp. 125-165). Nous confronterons ainsi l'offre numérique développée par la chaîne, hors téléphonie mobile, aux discours stratégiques des acteurs du groupe.

\section{SERVICE PUBLIC DE L'AUDIOVISUEL ET LOGIQUES DU WEB DYNAMIQUE}

Le Contrat d'Objectifs et de Moyens de France Télévisions fixe à ses chaînes l'impératif d'un développement complet de leurs offres numériques. La Charte des Antennes insiste sur la délinéarisation des programmes de la société publique qui doit désormais veiller à «(...) exploiter les possibilités offertes par la technologie numérique, en matière de format, de qualité d'image et de son, et de distribution et développer une offre de services de communication au public en ligne. »(Pflimlin, 2013 : 15-16). Chaque citoyen doit ainsi pouvoir accéder à l'audiovisuel public, quels que soient ses moyens ou sa situation géographique. La convergence technologique est devenue un moyen d'assurer le rayonnement des contenus audiovisuels publics, et ce grâce aux services de vidéo à la demande et aux supports numériques qui en assurent l'exposition, la prolongation et l'enrichissement par « (...) des informations pratiques sur les sujets traités et des références bibliographiques ou des renvois à des documents de toute nature permettant aux téléspectateurs de compléter leurs informations» (Cahier des charges de France Télévisions, 2009 : 11). Ainsi, le numérique apparaît comme un outil efficace permettant d'entretenir avec le public une relation privilégiée par «(...) l'utilisation de toutes les techniques de l'interactivité »(Pflimlin, 2013: 15-16). Il est d'ailleurs depuis plusieurs années au cœur des stratégies développées par les chaînes audiovisuelles publiques européennes, favorisant, d'après leurs dirigeants, le principe de l'égalité d'accès aux offres culturelles (sur cette question, cf. colloque Les stratégies sur le numérique et Internet, Lille, 1920 juillet 2000, [en ligne] http://www.culture.gouv.fr/culture/europe/lille/ tableronde2.htm).

\footnotetext{
${ }^{1}$ Le site web de France 2 étant similaire à ceux des autres chaînes du groupe France Télévisions, en particulier à ceux des chaînes France 3, France 4, France 5 - le site de France Ô se démarquant par une éditorialisation plus forte axée autour de l'information liée à l'outre-mer - nous choisissons d'examiner en détail celui de la chaîne la plus généraliste du groupe, qui s'affiche également comme la vitrine de cette marque-ombrelle. Les stratégies déployées sur les réseaux socio-numériques par les chaînes du groupe sont similaires, bien que ces dernières visent des publics différents et déploient à chaque fois une ligne éditoriale en cohérence avec les obligations fixées par leur cahier des charges.
} 
L'égal accès de tous aux offres culturelles numériques de l'audiovisuel public est l'un des principaux enjeux soulignés par les rapports de Jean-Pierre Plancade (Plancade, 2013) et de Pierre Lescure (Lescure, 2013). Ce dernier souligne «l'opportunité inédite » offerte par le développement des offres numériques qui permettent la dématérialisation des biens culturels, « (...) le partage et la découverte de nouvelles ouvres, de nouveaux artistes, de nouvelles esthétiques" (Lescure, $2013: 6$ ). Ce postulat, que l'on retrouve dans nombre de documents questionnant les missions du service public de l'audiovisuel à l'ère du numérique, est également au cœur des discours véhiculés par les pionniers du web dynamique qui placent le collectif au cœur de la mise en visibilité des informations, privilégiant le partage des biens numériques et les citations mutuelles, "sans autorité centrale» (Cardon, 2010: 77). Mais ces idéaux fondateurs, a priori propices au développement des offres culturelles numériques, sont non seulement menacés par l'affrontement entre les logiques du web et celles des institutions de l'espace public traditionnel, comme les médias audiovisuels, qui les utilisent à des fins plus stratégiques, mais mettent également au jour l'obsolescence des systèmes législatifs et économiques qui encadrent aujourd'hui le service public de l'audiovisuel en France, comparé à ses voisins européens.

La BBC, par exemple, est en Europe l'une des chaînes publiques ayant le mieux réussi à fédérer les citoyens britanniques autour de ses contenus en ligne, attirant chaque mois sur son site internet quelques 108 millions de visiteurs, soit $37 \%$ de la population du pays (Lherm, Charpentier, 2011: 40). À titre de comparaison, Médiamétrie situe le groupe France Télévisions dans le Top 30 Médiamétrie//NetRatings des sites les plus visités en France, mais en deçà des chiffres atteints par son homologue britannique: «France Télévisions se classe en 23è position et confirme sa position de leader des marques audiovisuelles sur le web, avec une audience record de 10,770 millions de visiteurs uniques. Une performance qui permet au groupe audiovisuel public de devancer nettement ses principaux concurrents TF1-WAT (34è), Canal + (66è) et M6 (107è)» (Sources : Médiamétrie//NetRatings, mai 2013). Les offres en ligne de France 2 paraissent minimes au regard de celles offertes sur le site de la BBC, qui déploie un nombre impressionnant de programmes de flux et de stocks (notamment de fictions) en VàD. Ce produit d'appel, associé aux différents tutoriels offerts par la chaîne britannique aux internautes de son site (comme ceux permettant l'apprentissage de langues étrangères), attirent un nombre croissant de visiteurs.

Ce décalage entre les deux groupes est pour partie lié aux différences qui opposent leur mode de gouvernance et leur financement. Le modèle britannique tire ainsi l'essentiel de ses ressources financières de la redevance publique, mais aussi d'importantes recettes commerciales hors publicité. Ainsi, la redevance représente pour la BBC en 2013 un revenu de 3.826.529 euros contre 1.431.800 euros pour le groupe France Télévisions (source: Observatoire Européen de l'Audiovisuel, disponible en ligne sur le site du Sénat: http://www.senat.fr/rap/r04-413/r04-41370.html, consulté le 15/10/2013). Quant aux recettes commerciales, elles représentent pour la BBC, en octobre 2013, 1.321.416 euros, contre 123.500 euros pour le groupe France Télévisions (Ibid.). La BBC dispose ainsi d'un budget deux fois supérieur à celui du groupe France Télévisions, budget financé quasiment sans publicité avec des recettes commerciales représentant près du $23 \%$ des recettes globales contre $6 \%$ pour le groupe public français (Ibid.). Autant dire que la vente en ligne de produits dérivés comme les séries diffusées par la chaîne représentent une manne financière non négligeable. Au contraire, le secteur de l'audiovisuel en France propose une offre numérique limitée par le principe de la chronologie des médias et concurrencée par l'émergence sur le marché de nouveaux acteurs qui «(...) échappent aux mécanismes 
nationaux de régulation et de financement» (Lescure, $2013: 5$ ). Les services de vidéo à la demande proposés par les chaînes publiques ou privées sont ainsi perçus par le public comme peu satisfaisants et trop chers face à l'offre gratuite - et souvent illégale - à laquelle ils ont désormais accès (Lescure, 2013 : 7-10). Le rapport Plancade souligne l'importance que les marques programmes vont prendre dans ce nouvel écosystème de consommation et par conséquent la nécessité de repenser l'environnement juridique qui permettra de pérenniser le financement des programmes et leur migration vers le numérique. De récentes mesures législatives vont d'ailleurs en ce sens, comme l'adoption par le Sénat d'un amendement modifiant la relation producteur-diffuseur ${ }^{2}$.

En outre, France Télévisions doit faire face tous les 5 ans au remaniement de ses contrats d'objectifs et de moyens, feuille de route signée avec l'Etat qui fragilise la pérennité des actions entreprises par les dirigeants du groupe public, dont la nomination est aussi dépendante des changements de gouvernements, contrairement à ses homologues britanniques, comme le souligne Mark Thompson, PDG de la BBC: « (...) La planification de notre budget confère à la BBC une autonomie qu'aucun responsable politique ne se risquerait à remettre en question : ce contrat décennal passé avec l'Etat est une solide garantie d'indépendance. Or cette convention, passée entre la BBC et son public, est un bien si précieux pour les Britanniques que le Parlement, qui peut intervenir à chaque instant, n'y a jamais touché. Jamais ! Quels que soient la majorité en place et les bouleversements politiques. $»^{3}$

En somme, France Télévisions doit aujourd'hui répondre à de nouveaux défis, dans un cadre législatif et politique mouvant. Repenser le «design» de l'action publique qui conserve une gouvernance dont l'architecture à été construite dans les années 1980 et qui se trouve aujourd'hui déstabilisée par le numérique semble urgent. Dans ce contexte, de quels moyens France 2, chaîne vitrine du groupe audiovisuel public, dispose-t-elle pour déployer ses offres numériques ? Les services proposés aux internautes répondent-ils véritablement aux contraintes imposées au service public de l'audiovisuel, aux logiques de la diversité, du pluralisme et de l'égalité d'accès aux biens culturels ?

\section{L'EXTENSION DE LA MARQUE FRANCE 2 A L'ECOSYSTEME WeB}

La proximité avec le public, au même titre que la relation client, sont désormais les objectifs prioritaires des community managers ${ }^{4}$ des chaînes télévisées, qui souhaitent transformer un média traditionnel en média conversationnel. Depuis 2009, France

${ }^{4}$ «Le Community Manager est l'acteur incontournable de la e-réputation, il est animateur, responsable des relations publiques dans les réseaux sociaux et veilleur. Le Community Manager est le «GO » de la communauté, celui qui anime, qui nourrit la conversation, qui crée des événements, qui répond aux questions, qui valorise les contributions de la communauté. [...] Le Community Manager est aussi celui qui développe des relations durables avec les consommateurs et qui gère les liens virtuels dans une stratégie de référencement [...]. Le Community Manager est enfin le gardien de l'identité numérique celui qui surveille et qui détecte les signaux positifs et négatifs. », Cadel, Peggy (2010, p. 111)
} 
Télévisions développe une offre numérique globale, lançant «(...) Pluzz.fr et Pluzz sur Orange et Free. De même, le portail info du groupe, France Télévisions Infos, regroupe l'ensemble de l'offre nationale, régionale et locale d'information de France Télévisions. »(CSA, 2010) Les comptes ouverts par France Télévisions sur Twitter servent à relayer sur les réseaux certains événements ponctuels. Lors de la finale du Top 14 de Rugby, ou du tournoi de Roland Garros, Myriam L'Aouffir, community manager du groupe, a ouvert avec son équipe des comptes spécifiques sur le site afin de diffuser des directs de ces programmes sportifs. Outre la création de blogs, comptes Twitter et Facebook, s'ajoutent des opérations transmédias, comme celle qui a permis de lancer des rencontres off line, en partenariat par exemple avec l'équipe de Taratata qui acceptait de faire visiter à ses «followers» les coulisses de l'émission musicale. Le livetweet permet enfin aux internautes d'entretenir une relation privilégiée avec certaines personnalités de l'audiovisuel, de prolonger des discussions commencées sur le petit écran, d'accéder aux coulisses des émissions, de bénéficier d'une expérience personnalisée.

Cette introduction des marques télévisuelles sur le web soulève toutefois plusieurs problèmes qui obligent les chaînes à développer des stratégies permettant aux téléspectateurs de retrouver sur Internet de nouveaux repères identificatoires (Leveneur, 2013). France Télévisions rassemble des chaînes dotées chacune d'une identité propre : $1^{\text {ère }}$, France 2, France 3, France 4, France 5 et France Ô, mais aussi diverses marques qui vont audelà des seules chaînes de diffusion : «(...) les marques chaînes, les marques animateurs, les marques programmes et les marques services $»^{5}$. Cette diversité pousse les webmestres de France 2 à faciliter avant tout la circulation des internautes entre les différents supports sites internet, comptes sur les réseaux socio-numériques, téléphonie mobile, VàD - qui se déploient autour des marques du groupe. Chaque interface possède en effet une logique énonciative qui lui est propre, à commencer par le site web de France 2, véritable vitrine de la chaîne et des valeurs qu'elle souhaite véhiculer auprès des internautes.

\section{France 2.fr : " Partagez, Dialoguez, Participez »}

Julia Bonaccorsi a montré de quelles façons un site web, en tant que dispositif sociotechnique, pouvait encadrer les échanges des internautes mais aussi déterminer en partie leurs usages (Bonaccorsi, 2013: 125-146). L'un des premiers points de l'analyse sémiologique de ce type de support concerne, d'après elle, les bords du texte à l'écran, qui déterminent son statut sémiotique et conditionnent la « circulation » des usagers sur le site.

On observe sur le site internet de France 2 une multiplicité de cadres et de bandeaux qui permettent de délimiter les différents menus accessibles. La page d'accueil affiche des textes succincts, composés essentiellement de titres et de propositions nominales ("Bientôt sur France $2 »$ ) ou de verbes permettant d'interpeller l'internaute («Voir et revoir », «A découvrir »)... Sur la page d'accueil, et sur les autres pages du site, les vidéos de la chaîne dominent sur le texte, France 2 souhaitant ainsi mettre en valeur ses contenus.

Le premier bandeau de la page d'accueil rappelle l'appartenance de France 2 au groupe France Télévisions, par le renvoi aux sites de ses différentes chaînes de diffusion (Voir ci dessous image 1). A la droite de celui-ci, plusieurs onglets renvoient à des marques spécifiquement développées pour le numérique: pluzz (VàD), FranceTVInfo, FranceTVSport et enfin un onglet «plus» qui renvoie à des sites partenaires comme

\footnotetext{
5 Interview de Miryam L'Aouffir datée du 24 mars 2011 et publiée sur le site www.blueboat.fr. URL: http://www.blueboat.fr/interview-de-cm-myriam-laouffir-groupe-france-televisions [consulté le 11/08/11].
} 
CultureBox, Geopolis, Monludo, etc. Ces derniers permettent aux internautes d'enrichir leurs connaissances géopolitiques et culturelles, d'accéder aux contenus enrichis des programmes jeunesse de la chaîne, ou encore de trouver sur le site "France TV Nouvelles Ecritures" des tutoriels interactifs qui conviennent à différents jeux didactiques sur la parité, le néojournalisme, le cinéma, etc.

Image 1 - Capture d'écran du haut de la page d'accueil sur site Internet de France 2, 25/08/13.

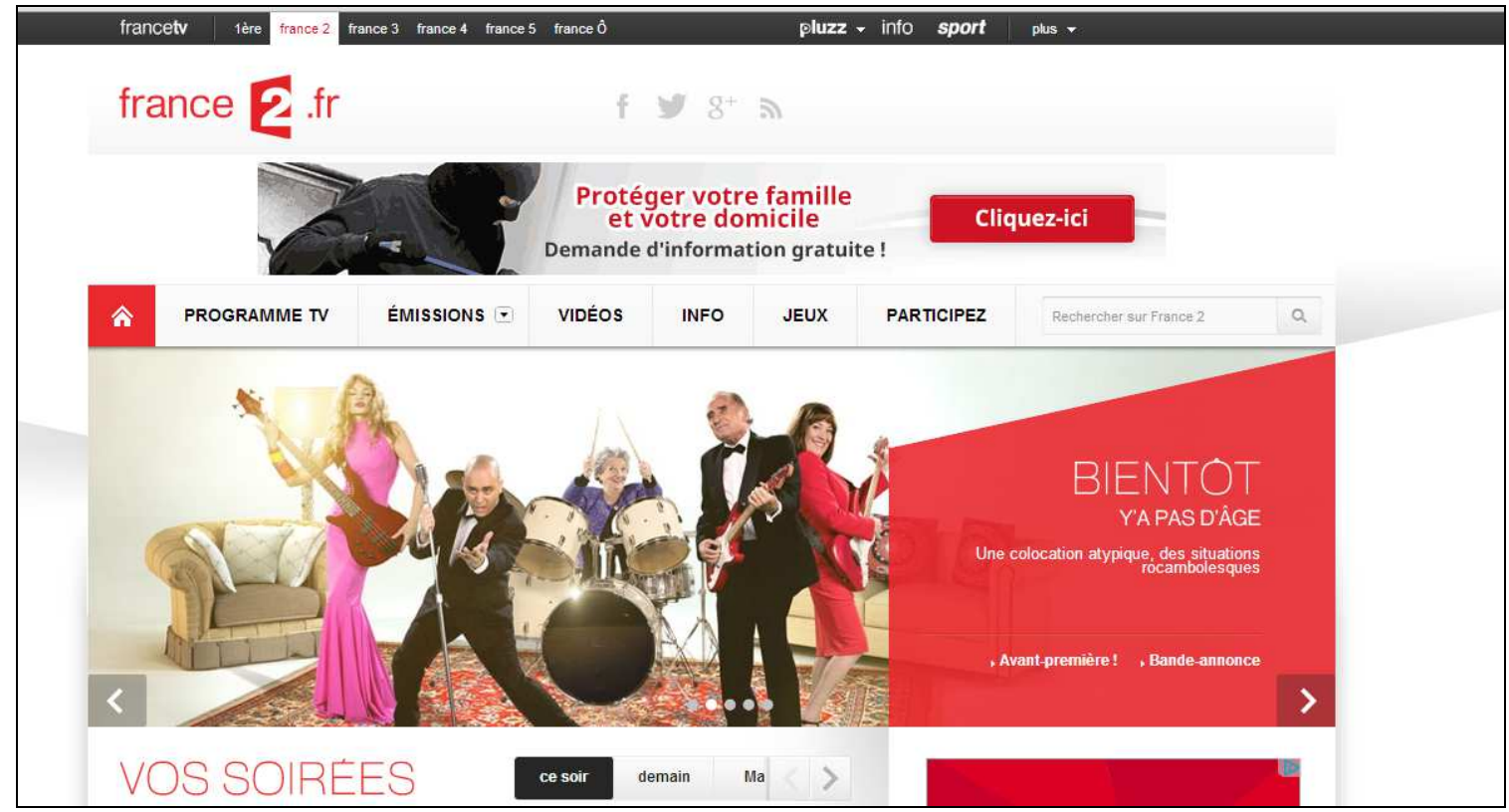

Le second bandeau met en valeur le logo de la chaîne, ainsi que ceux des réseaux socionumériques les plus emblématiques : Facebook, Twitter, Google+.

Cinq autres cadres se déroulent ensuite. Le premier, le plus visible, renvoie classiquement aux programmes que la chaîne souhaite promouvoir. Le suivant est consacré aux différents journaux télévisés de France 2, que l'internaute peut «voir et revoir » en ligne. Puis sont mises en valeurs les émissions emblématiques, les "marques filles" de la chaîne. Le bandeau suivant renvoie aux différents jeux en ligne dérivés de plusieurs émissions comme Motus ou Tout le monde veut prendre sa place. Enfin, un dernier cadre invite l'internaute à créer du lien avec la chaîne par une triple interpellation : "Partagez, dialoguez, contactez » (Voir ci-dessous image 2). Le premier onglet permet d'accéder à ses différents comptes sur les réseaux socio-numériques, le second renvoie aux forums de ses émissions ou invite à dialoguer avec ses animateurs ; le dernier verbe incite les téléspectateurs à prendre contact soit avec France 2, soit avec les médiateurs de l'information ou des programmes. Enfin en bas à droite de ce bandeau, l'internaute vigilant peut apercevoir un quatrième verbe : «participez» qui renvoie à une page détaillant ces différents espaces. En cliquant sur ce verbe (ou sur l'onglet homonyme dans le menu), le visiteur peut donner son avis sur les programmes de la chaîne, avoir davantage d'informations sur ses animateurs préférés (biographie, page contact, blogs éventuels), découvrir la synthèse des différents commentaires laissés par les autres internautes sur les fils Twitter ou Facebook, accéder aux actions de «la communauté » sur la toile ou enfin savoir quelle vidéo de France 2 crée le plus de «buzz» sur le net. 
Image 2- Milieu de la page d'accueil du site internet de France 2 le 25/08/13

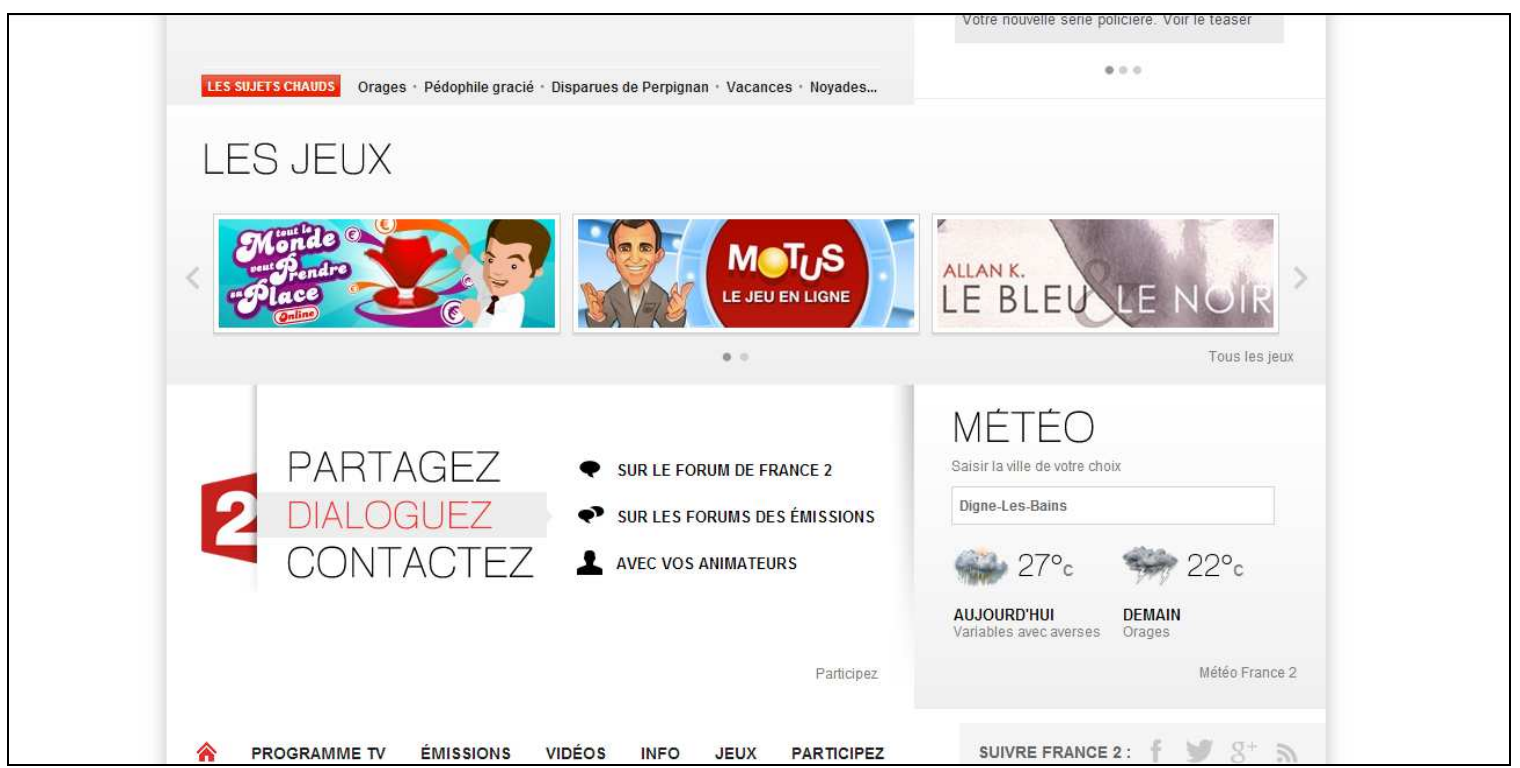

Le site web de France 2 se présente donc de prime abord comme une vitrine fidèle de la chaîne publique. Cet espace permet à l'usager d'accéder à différents contenus enrichis, comme l'illustre le site dédié à l'émission Télé-Matin sur lequel on peut visualiser les dernières chroniques du magazine, écouter la playlist de l'émission du jour, obtenir davantage d'informations sur les sujets traités par les chroniqueurs grâce aux renvois vers des sites spécialisés.

On remarque dès la page d'accueil que la marque France 2 place l'internaute au cœur du dispositif et l'invite à s'approprier les contenus de la chaîne : les infinitifs ou les impératifs utilisés pour inciter les visiteurs à cliquer renvoient rarement à la chaîne en tant que sujet. Le vouvoiement souvent utilisé ("vos soirées », "vos programmes ») fait écho à une idée commune aux supports numériques développés par plusieurs chaînes télévisés, publiques ou privées : internet développe un nouvel écosystème de consommation au sein duquel l'usager doit retrouver une offre personnalisée et différenciée (Voir Leveneur : 2013). Tant et si bien que le site laisse l'impression que l'internaute est libre de naviguer et de consulter les contenus désirés, alors même que l'agencement des différents cadres oriente très fortement la circulation de ce dernier. Cette impression est d'ailleurs renforcée par le fait que les internautes sont partie prenante de la mise en visibilité des contenus affichés.

\section{L'usager au coeur des stratégies de mise en visibilité.}

Sur le web et en particulier depuis l'arrivée des réseaux socio-numériques, l'éditorialisation du texte laisse place à un phénomène proche des logiques traditionnelles de l'audience. L'internaute est incité à aller vers les contenus les plus commentés, les plus vus. Cette logique de circulation énonciative est également à l'œuvre sur le site de France 2, puisque sur chaque page dédiée à un programme, outre les informations complémentaires accessibles en ligne, différents cadres mettent en valeur tantôt les sujets les plus commentés ( Les sujets chauds»), tantôt les vidéos les plus consultées, ou alors les derniers commentaires postés sur les comptes Facebook ou Twitter de la chaîne publique.

France 2 reprend à son compte la logique des sites de partages classiques comme Dailymotion ou Youtube. L'un des principes fondateurs d'Internet réside dans le fait que les 
internautes sont théoriquement libres de mettre en visibilité les informations qui les intéressent, hiérarchisant les informations selon une logique d'audience: "Même si ces hiérarchies peuvent faire l'objet de déformations stratégiques, elles reposent sur une évaluation collective conduite sans concertation préalable par les internautes eux-mêmes. » (Cardon, $2010: 50$ ). Les architectes du site web de France 2 n'ont fait que reprendre à leur compte ce principe intrinsèque, et ce d'autant plus aisément qu'il correspond à une logique télévisuelle ancienne, comme l'a souligné François Jost: «(..) la logique de classement est celle de toute chaîne de télévision [...]. Quant aux vidéos mises en ligne, elles sont en très grande majorité issues de chaînes de télévision, une toute petite partie d'internautes envoyant ses propres documents. Cette hiérarchisation des documents accessibles sur ces sites laisse perplexe quant à l'autonomie supposée de l'internaute, qui deviendrait une sorte de téléspectateur nomade. Force est de constater qu'il retrouve vite ses réflexes de téléspectateur, qui le poussent à aller vers les documents les plus fédérateurs. » (Jost, $2011: 26$ ).

Dominique Cardon souligne qu'à ses origines, le web se distinguait des médias traditionnels en développant une économie qui s'organisait autour d'un nombre de mesures variées; la réputation d'un document étant ainsi calculée en dénombrant l'ensemble des liens hypertextes que lui adressent d'autres documents du web (Cardon, 2013). Aujourd'hui, l'attention est calculée grâce au nombre de «like » ou de « retweets » qu'une page du web suscite. Comme le souligne le sociologue, dans le premier cas, la réputation est le résultat «(...) non intentionnel d'une évaluation de la qualité documentaire des informations, et dans le deuxième cas, la conséquence recherchée d'une action stratégique du producteur d'information pour susciter des gestes d'approbation » (Cardon, 2013). France 2 s'adapte tout naturellement à cette nouvelle tendance du web, à cette économie «du like»(Cardon, 2013), qui vise à susciter chez l'usager l'approbation de ses contenus, tout en orchestrant par le dispositif socio-technique du site internet cette mise en visibilité. L'internaute actif est ainsi largement valorisé : lorsque l'on clique par exemple sur l'onglet «activités de la communauté », puis sur une des vidéos à la une, l'on peut retrouver une page consacrée à l'émission en question, et différents onglets qui nous invitent à "réagir», "commenter» ou «en savoir plus» sur le programme. Le commentaire permet ensuite d'accéder à un fil de discussion constitué par les différentes remarques laissées par les internautes sur le programme. Les discussions les plus populaires sont mises en avant, les sujets qui suscitent le plus de commentaires tiennent le haut de la page et à droite de ces derniers les internautes les plus « actifs » sont valorisés.

En somme, du point de vue sémiotique, l'internaute est doublement mis en avant sur le site internet de France 2: convié à s'approprier les contenus, il a aussi la possibilité d'influencer la hiérarchisation des informations mises en ligne. Mais cette observation montre également que le site internet de France 2 ne présente pas de véritable logique éditoriale. Or, comme le souligne le rapport Lescure, la prescription et l'éditorialisation sont les conditions sine qua non, dans un univers numérique caractérisée par une hyper offre, de l'accès du public à la diversité culturelle et à la mise en valeur de la création française (Lescure, 2013 : 10). Le site web de France 2 est loin de cette logique, privilégiant des méthodes de classements qui ne lui permettent pas de se démarquer ni des autres acteurs culturels présents sur le web, ni même des chaînes télévisées privées. Ainsi, comme le souligne Jacques Chevallier, l'usager devient le "référent ultime " des services publics à l'orée du XXIème siècle (Chevallier, 2007), et le web 2.0 est particulièrement propice au renforcement de cette tendance, surtout depuis le développement de réseaux socionumériques. 


\section{"Suivre et aimer » France 2.}

Les renvois à Twitter et Facebook sur le site internet de France 2 sont systématiques, chaque page, chaque rubrique facilite l'accès aux différents comptes ou aux actualités de la chaîne. Plusieurs fenêtres de synthèses permettent aux internautes de suivre en direct les différents fils de discussions ou commentaires du jour.

Du point de vue quantitatif, si l'on s'en tient aux profils officiels de la chaîne, on relève, en août 2013, 446000 abonnés pour son compte Twitter, et 373000 «j'aime » sur sa page Facebook. Ces chiffres sont nettement inférieurs à ceux obtenus par les comptes de sa concurrente TF1 qui comptait à cette même période 756000 abonnés sur Twitter et 1,8 millions de fans pour sa page Facebook (chiffres d'ailleurs mis en valeur sur le site web de la chaîne). L'équipe digitale de TF1 a en effet développé depuis 2009 de nombreuses stratégies destinées à multiplier ses abonnés sur les réseaux socio-numériques, ces derniers constituant pour la régie publicitaire du groupe un produit d'appel non négligeable pour les nouveaux services VàD (Leveneur, 2013).

Du point de vue qualitatif, les commentaires publiés sur Facebook ou Twitter sont sensiblement les mêmes, du moins lorsqu'ils émanent des community managers de la chaîne. Il s'agit de valoriser les actualités liés aux programmes du jour, de lancer des fils de discussions sur différents thèmes liés aux grilles des programmes, de renvoyer au site web de la chaîne ou aux sites dédiés aux animateurs ou aux programmes.

Si l'on observe les fils de discussions consacrés aux programmes de France 2 sur Twitter, on note différents types d'interventions. Celui qui est par exemple consacré sur Twitter à la série «Fais pas ci, fais pas ça " (\#FPCFPC) affiche des commentaires liés à la diffusion d'un épisode en direct ; les internautes se plaisant à citer les phrases cultes des personnages ou à commenter leurs actions, le tout sur un registre essentiellement laudatif ou humoristique. France 2, comme d'autres chaînes de télévision, lance régulièrement des fils de discussions autour de ses programmes, avant ou pendant leur diffusion. En mars 2013, la chaîne a également expérimenté autour de la série Les Petits meurtres d'Agatha Christie une enquête interactive à laquelle les internautes pouvaient participer en ligne, tout en suivant un fil de discussion Twitter sur lequel ils donnaient leurs impressions sur le dispositif et échangeaient entre eux sur les indices fournis par la chaîne. L'observation de ces fils de discussion réalisée lors de la diffusion de l'épisode laisse apparaître des commentaires sur les acteurs, l'enquête, mais surtout le désarroi de nombreux internautes face à la complexité de suivre simultanément l'enquête à la télévision tout en répondant aux questions posées en ligne et en direct. Si l'expérience a montré ses limites, elle ouvre aussi de nouvelles perspectives pour la chaîne qui souhaite renouveler son public et rajeunir son audience: "À chaque fois que la télévision sait créer un événement dans le flux et le relayer par internet, elle ramène des jeunes à elle. »(Jost, 2011 : 30). Le principe de la télévision cérémonielle est ici renforcé par l'interactivité d'Internet et les réseaux socionumériques fédèrent les téléspectateurs autour d'un même événement, renforçant l'esprit communautaire. La notion de "fil de discussion" prend toutefois sur Twitter un sens restreint, le dispositif limitant le nombre de caractères écrits, on observe peu d'interpellations entre les internautes. Les commentaires critiques et les échanges véritables entre les utilisateurs sont davantage visibles dans les fils de discussions libres, en dehors de ceux lancés par les community managers de France 2.

Facebook et Twitter servent essentiellement à relayer les informations du site Internet de la chaîne publique, à susciter les commentaires des internautes qui sont conviés à "suivre et aimer » France 2, même si le registre euphorisant du «like»sur Facebook n'empêche nullement les commentaires acerbes de certains téléspectateurs. En effet, La page 
Facebook de la chaîne laisse apparaître plus clairement ce que l'on peut nommer une discussion entre les internautes ; les commentaires laissés par ces derniers, tantôt laudatifs, tantôt critiques, n'étant pas limités dans leur longueur, l'usager semble plus libre d'exprimer un point de vue. Reste que ces commentaires ou simples réactions sur ces deux réseaux sociaux numériques répondent à nouveau au diktat de l'audience, l'importance d'une information postée étant mesurée à l'aune des réactions qu'elle suscite.

On remarque en somme que ces dispositifs socio-techniques ont tendance à mettre tous les acteurs sur le même plan; les chaînes de télévision ont adopté les valeurs du web dynamique, ce que Sébastien Rouquette nomme les «cadres d'interprétations des utilisations légitimes du web» (Rouquette, 2009), véhiculés par les experts, la presse spécialisée et les essayistes. Or les réseaux socio-numériques répondent essentiellement à ce que d'aucuns qualifient à juste titre «d'artefacts de conversation » (Marti de Montety, 2012), contrairement aux forums qui semblent être des lieux d'échanges plus propices au débat public.

\section{Les forums de discussion : véritable lieu du débat public?}

Le service public se doit d'être «vecteur de lien social ». Cette expression, aussi labile que celle de «service public », peut s'entendre de différentes façons : agent de redistribution, le service public « (...) a pour fonction de mettre un certain nombre de biens à la portée de tous ; son action s'adresse de manière préférentielle aux plus démunis. »(Chevallier, 2007 : 19). L'on peut aussi entendre le lien social comme la possibilité offerte aux usagers de s'emparer des services publics, d'en discuter, d'autant plus que le recentrage sur l'usager constaté par Jacques Chevallier repose sur l'exigence de qualité dont il est souvent l'ultime référent, comme en témoigne la longue histoire de mesure de la qualité des programmes à la télévision française.

Or les offres web de France 2 s'articulent principalement autour de l'interpellation de l'internaute, convié à réagir, commenter, participer aux discussions sur les forums, les réseaux socio-numériques, à débattre des programmes diffusés par la chaîne, à poser des questions et à interpeller les médiateurs. Nous avons pu constater que les réseaux socionumériques offraient un espace somme toute restreint de discussion, se réduisant la plupart du temps à des commentaires relativement courts, parfois critiques. Les véritables lieux de l'argumentation sur le net restent les forums.

Ceux de France 2 révèlent des discussions extrêmement variées, parfois très longues et étayées entre les internautes. Les forums consacrés tantôt aux programmes de la chaine, tantôt à des sujets abordés dans les différents magazines ou journaux télévisés qu'elle produit, font se mêler des points de vue bruts et des expertises très argumentées. Certains internautes deviennent de véritables experts des programmes, se substituant à la chaîne, comme "Ondana », qui répond aux différentes questions posées par les internautes sur la série Meurtre au Paradis, que ce soit sur le scénario, le lieu de tournage, ou les prochaines diffusions. Les échanges se font sur un registre cordial, et la plupart du temps par vouvoiement.

Le forum le plus commenté reste celui du journal télévisé de 20 heures, où l'on recense entre janvier 2006 et août 2013, 1182 pages de discussions, sur des sujets extrêmement divers. Là aussi, les points de vue bruts et les expertises se mêlent, et les sujets les plus commentés sont ceux qui font la part belle à la polémique, comme lorsqu'un internaute reprend à son compte la théorie du complot à propos des attentats du 11 septembre 2001, suscitant alors 110000 réponses et 194000 lectures. Le forum est évidemment le lieu des réclamations en tous genres, mais on peut également y observer des échanges souvent 
maîtrisés, mobilisant parfois des connaissances très précises. Sur celui de l'émission «Secrets d'histoire», nombre d'internautes réagissent aux sujets diffusés, apportant des précisions sur les personnages ou les lieux historiques évoqués, et donnant leur opinion sur les sujets qu'ils souhaiteraient voir aborder dans le magazine grâce à un sondage proposé par la chaîne.

Les internautes de France 2, s'ils s'interpellent entre eux, rejettent toute forme de familiarité, répondant ainsi à la charte d'utilisation de la chaîne, même si les sujets polémiques sont ceux qui provoquent le plus de réactions, de même que les productions des professionnels de la chaîne (journalistes, animateurs, producteurs) dont les internautes commentent régulièrement le travail. Notons d'ailleurs que c'est principalement lorsque les posts concernent le travail des professionnels de France 2 que la notion de service public apparaît dans les échanges, comme en janvier 2006, lorsqu'un internaute au pseudonyme évocateur (Marre1) poste un sujet portant sur le traitement des grèves à New York. Quant aux interventions les plus lues, il s'agit de celles qui déploient le registre de la polémique ou de celles qui apportent aux lecteurs des connaissances complémentaires sur certains sujets, comme lorsqu'un téléspectateur revient sur l'histoire d'Israël et suscite par son texte circonstancié et didactique près de 12000 réponses et 261000 vues entre septembre 2006 et août 2013. Il est en outre très facile pour l'internaute qui s'identifie sur le forum d'avoir accès à une synthèse lui permettant de voir les derniers commentaires publiés sur le site et les réponses suscitées par ses propres interventions.

Bien que les échanges sur ces différents forums aient lieu au sein même d'un dispositif sociotechnique géré et modéré par France 2, force est de constater que les internautes gardent une distance critique très nette vis-à-vis de la chaîne publique, apportant des éclairages nouveaux sur les sujets traités, dialoguant de façon parfois experte entre eux sans qu'apparaissent des liens d'interconnaissance dans les échanges. De toute évidence, ces derniers n'ont nullement besoin que la chaîne publique déploie des forums pour débattre de ses programmes, puisque le web compte bon nombre d'autres lieux d'échanges. Mais de toute évidence, le label «France 2 » apporte aux discussions la garantie d'un débat dans le respect de la "netiquette », comme l'ont constaté par ailleurs deux chercheuses lors d'une étude comparative des forums dédiés à l'émission Le Jeu de la mort sur les sites de France 2 et du Post (Jouet, Le Caroff, 2013 : 158).

Toutefois, la disparité entre le nombre de commentaires postés et le nombre de lectures (ces dernières étant plus nombreuses) révèle que les internautes les plus actifs sont minoritaires au regard de la majorité silencieuse. L'égalité d'accès aux ressources numériques n'est pas totale, d'autant moins que nombre d'auteurs ont souligné l'importance des savoirs nécessaires à la bonne utilisation du web dynamique: "Qui s'aperçoit en effet que toute invitation à la participation active contient en elle-même la disqualification des silencieux et des passifs ? Derrière l'horizon démocratique du «toutparticipatif " se reproduisent des partages qui ont pour origine l'inégale distribution des capitaux socioculturels.» (Cardon, 2010: 80). Les éditeurs du logiciel de navigation Mozilla ont ainsi récemment publié un référentiel des compétences nécessaires à la bonne utilisation du web permettant aux internautes de mieux lire, écrire et collaborer dans l'univers numérique ${ }^{6}$.

\footnotetext{
${ }^{6}$ Référentiel disponible sur le site Webmaker : https://webmaker.org/fr/standard
} 


\section{CONCLUSION}

Le site internet de France 2 véhicule plusieurs valeurs qui ne sont pas propres à la chaîne publique, mais qui sont celles des pionniers du web, à savoir l'égalité d'accès aux ressources numériques, et l'appropriation des biens diffusés sur le web par les usagers eux-mêmes.

Si certaines pages du site apportent aux citoyens des informations complémentaires qui enrichissent les programmes de la chaîne, ces dernières semblent relativement limitées au regard des questionnements des internautes, visibles sur les forums et les réseaux socionumériques. Au final, France 2 se positionne sur la toile comme un agent facilitateur de lien et d'échanges, plutôt que comme un fournisseur de contenus allant, comme ceux de la BBC, au-delà de ses programmes. Il faut que l'internaute aille chercher les informations sur les sites partenaires comme Géopolis ou France TV Nouvelles Ecritures pour trouver des offres interactives et particulièrement enrichies qui répondent notamment aux principes du pluralisme et de la diversité.

Certes, nombre de contraintes pèsent aujourd'hui sur l'audiovisuel public, qui doit de plus en plus compter sur des opérateurs privées pour assurer ses missions, notamment s'agissant de la production audiovisuelle, majoritairement externalisée, ce qui ne permet pas au groupe public de disposer de la totalité des droits sur ses contenus (Voir Regourd, 2011), et par conséquent de les rendre complètement accessibles sur le web. Aujourd'hui soumis aux lois du marché et de la concurrence, répondant aux mêmes principes d'efficacité que les entreprises privées, le service public de l'audiovisuel doit en outre se substituer aux mécanismes d'auto-régulation sociale, assurer la cohésion sociale et un égal accès aux biens culturels communs (Chevallier, $2007: 18$ ). Le web dynamique charrie avec lui des valeurs propices au déploiement de ces missions, mais encore faut-il que les groupes audiovisuels publics français disposent des moyens - financiers et juridiques - de les assurer.

Au-delà de ces contraintes, France 2 n'affiche pas de positionnement clair sur le web 2.0. $\mathrm{Au}$ final, les marques " programmes » l'emportent sur la marque chaîne, la fiction ou les jeux apparaissant comme les produits culturels phares du numérique. La chaîne de diffusion ne fait qu'adopter les valeurs communes du web dynamique, et se plier à une logique de l'audience alors même que l'importance de la prescription et de l'éditorialisation sur le numérique ont été soulignées par ailleurs (Lescure, 2013:10). Pour l'heure, le groupe audiovisuel public n'affirme pas suffisamment clairement un positionnement qui permettrait à l'internaute "moyen» de mieux identifier son engagement dans la promotion de la diversité culturelle et la création. 


\section{REFERENCES BIBLIOGRAPHIQUES}

Barats, Christine (2013), Manuel d'analyse du web, Paris : Armand Colin.

Bonaccorsi, Julia (2013), "Approches sémiologiques du web", (p.125-146), in Barats, Christine (dir.), Manuel d'analyse du web, Paris : Armand Colin.

Cadel, Peggy (2010) «Le marché de l'e-réputation », Les Cahiers du numérique 4/2010 (Vol. 6), p. 111-121.

Cailler, Bruno (2011), "Quel futur pour la programmation de télévision : le danger du « tout consultatif »?, ESSACHESS. Journal for communication studies, vol. 4, p. 129-139.

Cardon Dominique (2010), La démocratie Internet. Promesses et limites. Paris : Seuil, coll. « La République des idées ».

Cardon Dominique (2013), « «Du lien au like» deux mesures de la réputation sur Internet ", Communication présentée au séminaire de l'Ecole Thématique Identité Numérique, Sète : CNRS, 30 juin-5 juillet 2013.

Chevallier, Jacques (2007), «Les nouvelles frontières du service public », Regards croisés sur l'économie, $\mathrm{N}^{\circ}$ 2, p.14-24.

Jost, François (2010), "Culture et dépendances. Les avatars d'une mission de service public », Le Temps des médias, $\mathrm{n}^{\circ} 14$, pp.219-231

Jost, François (2011) « Où va la télévision ? De l'influence de la télévision numérique sur les programmes et la programmation. »(p.23-37), in Delavaud, Gilles (Dir.), Permanences de la télévision, Paris : Editions Apogée.

Jouet, Josiane, Le Caroff, Coralie (2013) " L'observation ethnographique en ligne", (p.147-165) in Barats, Christine (dir.), Manuel d'analyse du web, Paris : Armand Colin.

Laurent, Gilles, Marchandise, Jacques-François (2013) La dynamique d'Internet. Prospective 2030, Paris : Commissariat Général à la Prospective, coll. Etudes n ${ }^{\circ} 1$.

Lescure, Pierre (2013) "Contribution aux politiques culturelles à l'ère numérique », Mission «Acte II de l'exception culturelle », Mai 2013.

Leveneur, Laurence (2013), "Marques télévisuelles et réseaux sociaux : vers un renouvellement des stratégies identitaires des chaînes généralistes françaises ?" (p.163176), in Laurichesse Hélène (dir.), La stratégie de marque dans l'audiovisuel, Paris : Armand Colin.

Plancade, Jean-Pierre (2013), Rapport d'information fait au nom de la commission de la culture, de l'éducation et de la communication par le groupe de travail sur les relations entre le sproducteurs audiovisuels et les édietrus des services de télévision, Sénat, 30 mai 2013.

Regourd, Serge (2011), "Politiques culturelles et sidération technologique » (p. 5-15), in Internet : enjeux, Paris : Fondation Gabriel Péri.

Rouquette, Sébastien (2009), L'analyse des sites internet. Une radiographie du cyberespace. Paris, INA/De Boeck.

\section{Articles de presse}

Sophie Lherm, Olivier Charpentier (2011), «Pourquoi l'audiovisuel public n'aurait pas sa place sur le net? ", Télérama n $^{\circ} 3184,19$ janvier 2011, p.40. 
Renaud Revel (2011), "Rémy Pflimlin; "Nous devons toucher tous les publics" », L'express, 2 novembre 2011

Renaud Revel (2012), «BBC, ZDF, France Télé: regard croisé de 3 PDG», L'Express, 3/10/2012, disponible en ligne sur http://www.lexpress.fr/actualite/mediapeople/media/bbc-zdf-france-tele-comparaison-entre-trois-modelesdifferents 1169483.html

Voir «Audiovisuel : le Sénat a voté un amendement surprise modifiant la relation producteurs-diffuseurs ", Le Monde daté du 2/10/13, disponible en ligne sur http://www.lemonde.fr/actualite-medias/article/2013/10/02/audiovisuel-l-amendementsurprise-du-senat-qui-pourrait-irriter-les-producteurs 3488778 3236.html

\section{Webographie}

Cahier des charges de la société nationale de programme France Télévisions (2009), décret $\mathrm{n}^{\circ}$ 2009793, Paris : Journal officiel du 25 juin 2009 [en ligne] http://www.csa.fr/Television/Leschaines-de-television/Les-chaines-hertziennes-terrestres/Cahier-des-charges-de-France$\underline{\text { Televisions }}$

CSA (2010) Bilan France Télévision 2010, Paris : CSA [en ligne] www.csa.fr

Granier, Jean-Maxence, Patrin, Valérie (2009), «Marques médias et médias marque. Jeux de frontières", Séminaire Média de l'IREP, [en ligne] http://fr.slideshare.net/lpto/mediamarque-marquemedia-sminaire-irep-2009

Pflimlin, Rémy (2013), Charte des antennes de France Télévisions, [en ligne] http://www.francetelevisions.fr/downloads/charte des antennes web.pdf

Richard, Dominique (2006), Rapport d'information sur les missions du service public de l'audiovisuel et l'offre de programmes au nom de la Commission des affaires culturelles, Paris, $\mathrm{n}^{\circ} 2917$ [en ligne] http://www.assemblee-nationale.fr/12/rap-info/i2917.asp 